Revue d'histoire de l'Amérique française

Q4. REVUE D'HISTOIRE DE L'AMÉRIQUE FRANÇAISE

\title{
La CECM et la démocratisation du financement scolaire à Montréal (1963-1973)
}

\section{Jean-Philippe Croteau}

Volume 62, numéro 1, été 2008

URI : https://id.erudit.org/iderudit/029663ar

DOI : https://doi.org/10.7202/029663ar

Aller au sommaire du numéro

\section{Éditeur(s)}

Institut d'histoire de l'Amérique française

\section{ISSN}

0035-2357 (imprimé)

1492-1383 (numérique)

Découvrir la revue

\section{Citer cet article}

Croteau, J.-P. (2008). La CECM et la démocratisation du financement scolaire à Montréal (1963-1973). Revue d'histoire de l'Amérique française, 62(1), 5-34. https://doi.org/10.7202/029663ar
Résumé de l'article

Au début des années 1960, les dirigeants de la Commission des écoles catholiques de Montréal (CECM) dénoncent le faible développement de leur secteur secondaire qui accuse un important retard sur celui de la Protestant School Board of Greater Montreal (PSBGM). Le mode de répartition inéquitable de la taxe scolaire est perçu comme l'une des principales causes de ce retard qui fait obstacle à la scolarisation des francophones. À l'époque, le montant de la taxe scolaire, prélevée par cotisation sur les propriétés foncières, est réparti entre les écoles publiques d'après la croyance religieuse des propriétaires. En raison du niveau de richesse plus élevé des protestants, la PSBGM est favorisée par ce mode de financement. Cette étude vise à reconstituer les différentes étapes de la campagne de pression de la CECM pour obtenir l'abolition de ce mode de financement scolaire. Elle tend à montrer le caractère indissociable que prennent la question de la démocratisation de l'enseignement secondaire public et celle de la redistribution équitable des ressources financières des commissions scolaires montréalaises.
Tous droits réservés @ Institut d'histoire de l'Amérique française, 2009
Ce document est protégé par la loi sur le droit d'auteur. L'utilisation des services d'Érudit (y compris la reproduction) est assujettie à sa politique d'utilisation que vous pouvez consulter en ligne.

https://apropos.erudit.org/fr/usagers/politique-dutilisation/ 


\title{
La CECM et la démocratisation du financement scolaire à Montréal (I963-ı973)
}

\author{
Jean-Philippe Croteau \\ Département d'histoire \\ Université de Hearst
}

RÉsumÉ - Au début des années 1960, les dirigeants de la Commission des écoles catholiques de Montréal (CECM) dénoncent le faible développement de leur secteur secondaire qui accuse un important retard sur celui de la Protestant School Board of Greater Montreal (PSBGM). Le mode de répartition inéquitable de la taxe scolaire est perçu comme l'une des principales causes de ce retard qui fait obstacle à la scolarisation des francophones. À l'époque, le montant de la taxe scolaire, prélevée par cotisation sur les propriétés foncières, est réparti entre les écoles publiques d'après la croyance religieuse des propriétaires. En raison du niveau de richesse plus élevé des protestants, la PSBGM est favorisée par ce mode de financement. Cette étude vise à reconstituer les différentes étapes de la campagne de pression de la CECM pour obtenir l'abolition de ce mode de financement scolaire. Elle tend à montrer le caractère indissociable que prennent la question de la démocratisation de l'enseignement secondaire public et celle de la redistribution équitable des ressources financières des commissions scolaires montréalaises.

ABSTRACT - In the early 1960s, the leaders of the Montreal Catholic School Commission condemned the weak development of their secondary school programs, which were falling significantly behind those of the Protestant School Board of Greater Montreal (PSBGM). An unfair method of allocating school taxes was seen as one of the main reasons behind this lag, which represented a barrier to francophone education. At that time, school taxes were collected according to real estate assessments and were divided among the public schools

1. Cet article s'inspire de Jean-Philippe Croteau, Le financement des écoles publiques à Montréal (1869-1973): deux poids, deux mesures, thèse de doctorat (histoire), Université du Québec à Montréal, 2006. 
according to the religious affiliation of the property owners. Because of the higher level of wealth among Protestants, the PSBGM benefited from this funding model. This article aims to reconstruct the different stages in the CECM's lobbying campaign to abolish this model of school funding. It will illustrate the direct connection between the democratization of public secondary education and the equitable redistribution of financial resources among Montreal's school boards.

$\mathrm{D}$ ans l'historiographie récente, la réforme de l'éducation des années 1960 est souvent présentée comme un tournant émancipateur dans l’histoire de la société québécoise qui se libère à ce moment précis de ses attaches traditionalistes et entre de plain-pied dans la modernité. Les grands acteurs de cette trame historique sont les Québécoises et les Québécois qui participent à la reprise en main de leur destin collectif. Représentant l'incarnation de la volonté populaire, l'État parvient à mettre fin à des décennies - voire à un siècle - de retard social, économique et éducatif que la société québécoise accusait sur l'ensemble des sociétés nord-américaines. Au cœur de ce récit épique, on retrouve un enjeu fondamental, la démocratisation de l'éducation.

Aujourd'hui, quarante ans plus tard, ce portrait louangeur de la réforme éducative des années 1960 est de plus en plus contesté. On assiste, surtout depuis les dernières décennies, à une historiographie qui remet en question les acquis de la réforme éducative des années 1960. Malgré tout, il reste que les historiens, les sociologues et les éducateurs présentent un bilan de la réforme éducative des années 1960 qui pose un regard essentiellement institutionnel et centré surtout sur la figure emblématique de l’État. Ils retiennent principalement de cette période les mesures éclatantes qui ont suivi les recommandations de la Commission royale d'enquête sur l'éducation - plus connue sous le nom de Commission Parent - comme la création du ministère de l'Éducation, du Conseil supérieur de l'éducation, des cégeps et du réseau de l’Université du Québec. Ils évacuent, pour la plupart, l’aspect financier de la réforme de l'éducation, un aspect pourtant capital, si l'on veut comprendre les conditions qui ont permis à la collectivité québécoise de mettre fin au cycle de la sous-scolarisation et de l'infériorité économique des Canadiens français. Or, il nous apparaît que l'aspect financier demeure indissociable de la question de la démocratisation de l'éducation au Québec et mérite un traitement à part pour bien mesurer son impact sur la généralisation de l'éducation au sein des populations montréalaise et québécoise à partir des années 1960. 
Depuis la dernière moitié du $\mathrm{xIx}^{\mathrm{e}}$ siècle, la responsabilité du financement des écoles publiques reposait très largement sur le fardeau fiscal des communautés et surtout sur leur capacité à assumer les coûts des services éducatifs ${ }^{2}$. En effet, une proportion croissante des revenus des commissions scolaires provenait de la taxe scolaire prélevée par cotisation sur les propriétés foncières. Ainsi, l'offre des services éducatifs dépendait en grande partie du niveau de richesse des propriétaires d'une communauté. À Montréal, la situation était encore plus complexe. Les revenus de la taxe scolaire étaient répartis entre les écoles publiques, catholiques ou protestantes, d'après l'appartenance religieuse des propriétaires. Une formule qui a toujours désavantagé les catholiques, moins fortunés dans l'ensemble que les protestants et qui n’a pas permis à la Commission des écoles catholiques de Montréal (CECM) d'ériger un secteur secondaire pouvant soutenir la comparaison avec celui de la Protestant School Board of Greater Montreal (PSBGM).

C'est cette situation que la CECM souhaite corriger au début des années 1960 à la faveur d'un nouveau contexte politique. La CECM tient responsable le mode de partage de la taxe scolaire du faible développement de sa filière secondaire et de la sous-scolarisation des francophones. Elle lance une campagne de pression dès le début des années 1960 pour obtenir l'adoption d'un mode de financement scolaire plus équitable, afin que les francophones puissent bénéficier de l'égalité des chances avec leurs camarades protestants.

Cet article examine les grandes étapes de cette double démocratisation, celle du financement scolaire et celle de l'enseignement secondaire. À travers le discours réformiste des dirigeants de la CECM, nous montrerons le caractère indissociable que prennent la démocratisation de l'enseignement secondaire et la redistribution équitable des ressources financières des commissions scolaires montréalaises. D’après nous, la démocratisation du financement scolaire à Montréal a été un préalable essentiel à la démocratisation de l'enseignement secondaire et elle a constitué la condition nécessaire au «rattrapage scolaire» des francophones de la métropole.

Rappelons que la question du financement scolaire a occupé peu de place jusqu'à ce jour dans l'historiographie, si ce n'est pour dénoncer l’importance des dépenses engagées par l'État en éducation ${ }^{3}$. Si l’on

2. Environ $22 \%$ des sommes consacrées à l'éducation viennent de l'État en 1856 contre $11 \%$ en 1873, d'après Gérard Filteau et Lionel Allard, Un siècle au service de l'éducation, 1851-1951, 1: 1851-1911: l'inspection des écoles dans la province de Québec (s.l., s.d., 1955), 36.

3. Nous retenons toutefois une exception, l'article de Guy Rocher qui fait le bilan de la démocratisation de l'éducation au Québec à partir des années 1960 jusqu’à aujourd'hui. Il présente 
constate un intérêt croissant pour la question du financement de l'éducation, ces études se penchent surtout sur la période de la fin du $\mathrm{XIX}^{\mathrm{e}}$ siècle et de la première moitié du $\mathrm{xx}^{\mathrm{e}}$ siècle $\mathrm{e}^{4}$. Il est plutôt paradoxal que, malgré une surabondance de travaux sur la Révolution tranquille et la réforme Parent, les questions relatives au financement scolaire dans les années 1960 demeurent encore largement inexplorées. Cet article vise donc à combler ce quasi-silence historiographique en examinant un phénomène peu connu de la réforme éducative et même de la Révolution tranquille: la démocratisation du financement scolaire à Montréal.

Nous avons mené nos recherches surtout aux archives des commissions scolaires montréalaises, aujourd'hui la Commission scolaire de Montréal (CSDM) et de la English Montreal School Board (EMSB). Nous avons consulté principalement les rapports financiers et les rapports annuels qui renferment beaucoup de renseignements sur les budgets des commissions scolaires et les inscriptions des élèves. Nous avons aussi examiné les procèsverbaux des commissions scolaires montréalaises, les mémoires présentés aux commissions royales d'enquête et la correspondance des commissaires pour reconstituer les relations politiques entre les acteurs scolaires et le

brièvement la démocratisation du financement scolaire et prétend que ce fut sans doute l'une des réformes les plus faciles à réaliser grâce à la création en 1964 du ministère de l’Éducation. Dans notre article, nous ferons la démonstration que la démocratisation du financement a été un enjeu beaucoup plus complexe qu'il n'y paraît et qu'il a fallu bien des compromis entre les acteurs scolaires et le gouvernement provincial pour arriver à une entente. Voir Guy Rocher, «Le bilan du Rapport Parent: vers la démocratisation", Bulletin d'histoire politique, 12,2 (hiver 2004): 117-128.

4. Un certain nombre d'études relatent le partage des responsabilités fiscales entre l'État et les communautés locales. Elles font la démonstration que le fardeau fiscal incombe essentiellement aux parents et aux contribuables. À l'échelle du Québec, voir Ruby Heap, L'Église, l'État et l'enseignement primaire public catholique (1897-1920), thèse de doctorat (histoire), Université de Montréal, 1987, 297-343; Andrée Dufour, «Financement des écoles et scolarisation au Bas-Canada: une interaction État-communautés locales (1826-1859)", Historical Studies in Education/Revue d'histoire de l'éducation, 6,2 (automne 1994): 219-252; et J.-P. Charland, L'entreprise éducative au Québec, 1840-1900 (Sainte-Foy, Les Presses de l’Université Laval, 2000), 161-172. Pour le cas précis de Montréal, voir Terry Copp, Classe ouvrière et pauvreté. Les conditions de vie des travailleurs montréalais 1897-1929 (Montréal, Boréal Express, 1978), 61-75 ; Wendy Johnston, «Aux sources du développement inégal: le financement de l'enseignement public à Montréal de 1920 à 1945 ", Canadian Historical Review, LXXVI,1 (1995): 43-77; Robert Gagnon, Histoire de la Commission des écoles catholiques de Montréal. Le développement d'un réseau d'écoles publiques en milieu urbain (Montréal, Boréal, 1996), 400 p. et J.-P. Croteau, Le financement des écoles publiques..., op. cit., 452 p. Voir aussi pour l'éducation protestante, R. MacLeod et M. A. Poutanen, Meeting of the People. School Boards and Protestant Communities in Quebec, 1801-1998 (Montréal, McGill-Queen’s University Press, 2004), $350 \mathrm{p}$. Enfin, pour la question du développement scolaire d'une région spécifique, soit la Mauricie, voir Jocelyne Murray, Apprendre à lire et à compter. École et société en Mauricie (1850-1900) (Sillery, Septentrion, coll. "Cahiers des Amériques», 2003), 350 p. 
gouvernement québécois. Enfin, nous avons dépouillé les articles et les éditoriaux de la presse qui constituent un baromètre important pour mesurer les courants d'opinion parmi la population montréalaise.

\section{LE PACTE SCOLAIRE DE 1869}

La question du financement scolaire n'est pas contemporaine à la Révolution tranquille. Elle interpelle les acteurs de la sphère éducative dès la fondation, en 1846, de la Commission des écoles catholiques de Montréal (CECM) et de la Protestant School Board Commissioners of the City of Montreal (PBSCCM), qui deviendra plus tard, en 1951, la Protestant School Board of Greater Montreal (PSBGM) ${ }^{5}$. Dans la dernière moitié du XIX ${ }^{e}$ siècle et pendant plus d'un siècle, cette question constituera une pomme de discorde entre les dirigeants scolaires catholiques et protestants.

Dans les années 1840, les législateurs ne prévoient pas instaurer pour les villes de Québec et de Montréal une taxe scolaire sur les propriétés foncières comme ailleurs au Bas-Canada ${ }^{6}$. Les revenus de ces deux

5. Fondée en 1846, la Protestant Board of School Commissioners of the City of Montreal (PBSCCM) administre les écoles protestantes sur le territoire de la ville de Montréal. Entre 1846 et 1955, dix autres commissions scolaires protestantes sont créées sur l'île de Montréal. En 1925, pour remédier à la crise financière de certaines commissions scolaires, la Montreal Protestant Central School Board (MPCSB) est mise sur pied. Ses prérogatives sont essentiellement de nature financière : elle récupère les recettes de la taxe prélevée par les autorités municipales et les redistribue aux onze commissions scolaires sous sa juridiction d'après une formule de péréquation. Les commissions scolaires conservent toutefois une certaine latitude sur le plan administratif et pédagogique. Leur autonomie prend fin, en 1945, alors que neuf d'entre elles sont incorporées à la MPCSB, qui hérite de leurs compétences administratives. Bien qu'elles aient renoncé à leurs responsabilités administratives, les commissions scolaires locales continuent d'exister virtuellement puisqu'elles nomment leurs représentants à la MPCSB. En 1951, la MPCSB devient la Protestant School Board of Greater Montreal (PSBGM). Enfin, avec la loi 71, adoptée en 1973, toutes les commissions locales sont annexées à la PBSCCM qui est rebaptisée PSBGM. Pour éviter toute confusion, nous emploierons uniquement le terme PSBGM pour référer d'abord à la PBSCCM, puis à la nouvelle structure scolaire unifiée à partir de 1951. Fondée, elle aussi, en 1846, la commission scolaire catholique prend le nom cette même année de Bureau des commissaires catholiques romains de la cité de Montréal. En 1900, elle change de nom pour la Commission des écoles catholiques de Montréal (CECM). À des fins de simplification, nous n'utiliserons que le vocable CECM tout au long de cet article. Voir à ce sujet les ouvrages de R. Gagnon, Histoire de la Commission..., op. cit., 400 p. et R. MacLeod et M. A. Poutanen, Meeting of the People..., op. cit., $450 \mathrm{p}$.

6. Au Bas-Canada, à partir de 1841, le principe de la «contribution obligatoire» est instauré. Les propriétaires fonciers doivent élire des commissaires d'écoles pour un mandat de trois ans. Ces derniers sont chargés de prélever une taxe sur la valeur des propriétés foncières équivalant au montant des subventions gouvernementales. À Montréal et à Québec, les commissaires sont nommés par le Conseil de Ville. La loi ne prévoit pas de prélever une taxe scolaire. Le trésorier verse une subvention aux commissions scolaires à même le budget du Conseil de Ville. Ce n'est 
commissions scolaires sont basés sur les subventions gouvernementales, sur celles du Conseil de Ville et les droits de scolarité payés mensuellement par les parents des écoliers et des écolières. Ces revenus s'avèrent rapidement insuffisants face à la croissance de la population scolaire de la métropole.

Le premier ministre du Québec, Pierre-Joseph-Olivier Chauveau et ministre de l'Instruction publique depuis 1867 , souhaite remédier à cette situation. En mars 1869, son gouvernement dépose un projet de loi qui prévoit la création d'une taxe scolaire, à Montréal, prélevée sur les propriétés immobilières. Auparavant, il doit composer avec les commissaires catholiques et protestants qui ne s'entendent pas sur le mode de répartition de cette taxe. Les protestants réclament que les revenus versés aux écoles publiques soient divisés d'après la religion des propriétaires. Ceux-ci trouvent inacceptable d'être mis à contribution pour les écoles catholiques, des écoles qui enseignent une doctrine contraire à leurs convictions religieuses. Les catholiques, quant à eux, voient plutôt l'éducation comme un bien public. Les sommes consacrées à l'éducation devraient donc être partagées d'après le nombre d'enfants à instruire ${ }^{7}$.

Homme de compromis, Chauveau coupe la poire en deux. En effet, le premier ministre tient compte, dans le projet de loi, à la fois des revendications des protestants et des catholiques. Quatre listes sont dressées. Les propriétés des catholiques sont inscrites sur la première, les propriétés des protestants sur la seconde et celles des compagnies commerciales et des sociétés sur la troisième. Enfin, la quatrième liste comprend les propriétés des Églises qui bénéficient de l’immunité fiscale ${ }^{8}$.

qu'en 1869 qu'une taxe scolaire est prélevée sur les propriétés foncières dans les villes de Québec et Montréal. Voir R. Gagnon, Histoire de la Commission..., op. cit., 20-24, et J.-P. Charland, L'entreprise éducative au Québec..., op. cit., 55-72.

7. Débats à l'Assemblée législative du Québec, 3, 19 et 22 mars 1869. Voir l'une des premières études publiées sur le sujet, Keith D. Hunte, The Ministry of Public Instruction in Quebec, 1867-1875: A Historical Study, thèse de doctorat (histoire), Université McGill, 1964, 128-151. À propos du contexte dans lequel la Loi sur l'éducation a été adoptée, voir J.-P. Croteau, op. cit., 49-79. Voir aussi l'article de Jean-Philippe Croteau et Robert Gagnon, «Les débats sur le partage de la taxe scolaire à Montréal (1869-1899): enjeux et rivalités", Revue d'histoire de l'éducation/Historical Studies in Education, 20,1 (printemps 2008): 32-68. À propos des débats à l'Assemblée législative sur la Loi sur l'éducation en mars 1869, voir l'analyse de Marcel Hamelin, Les premières années du parlementarisme québécois (1867-1878) (Québec, Les Presses de l’Université Laval, coll. «Les cahiers d’histoire de l’Université Laval», 1974), 50-59. Sur la carrière de Pierre-Joseph-Olivier Chauveau, voir Hélène Sabourin, À l'école de P.-J.-O. Chauveau. Éducation et culture au XIX siècle (Montréal, Leméac, 2003), 230 p. ; Jean Hamelin et Pierre Poulin. «Pierre-Joseph-Olivier Chauveau », Dictionnaire biographique du Canada (Sainte-Foy, Les Presses de l'Université Laval, 1982), XI : 194-204.

8. "Loi sur l'éducation", Journal de l'éducation, XIII,4 (avril 1869): 50-51. 
Les sommes prélevées sur la liste des catholiques et sur celle des protestants sont versées respectivement aux commissions catholiques et protestantes. Les protestants ont donc remporté un gain majeur. Toutefois, ils essuient un refus du premier ministre Chauveau pour le partage des taxes des compagnies commerciales et des sociétés. Pour contenter la majorité catholique, le gouvernement Chauveau divise les taxes des propriétés commerciales et des sociétés - appelées communément "neutres» - au prorata des populations catholique et protestante plutôt que d'après la religion des propriétaires ou des actionnaires.

Bien que cette nouvelle source de revenus soit bien accueillie par les commissaires protestants et catholiques, son mode de répartition ne tarde pas à poser problème. En général plus fortunés que les catholiques, les protestants sont favorisés par ce mode de financement scolaire. Ainsi, en 1899 , bien que les protestants ne représentent que le quart de la population montréalaise, la PSBGM reçoit autant d'argent de la taxe scolaire que les catholiques. Une situation vigoureusement décriée par la CECM. Pendant l'année scolaire 1899-1900, la PSBGM dépense 19 \$ par élève et la CECM seulement 9 \$. Si l'écart se rétrécit entre les deux commissions scolaires au fil des décennies, l'inégalité persiste tout de même au début des années 1960. Dans les années 1950, la CECM dépense 285 pour chacun de ses élèves, tandis que la PSBGM consacre $352 \$$ par élève ${ }^{10}$.

Cette part généreuse de l'assiette fiscale permet à la PSBGM d'investir différents champs d'activités scolaires, dont l'enseignement secondaire. En 1870, elle acquiert une institution privée renommée, le High School of Montreal, et fonde cinq ans plus tard le High School for Girls. Dès la fin du XIX ${ }^{\mathrm{e}}$ siècle, les enfants protestants de la métropole bénéficient d'un accès à une filière secondaire qui conduit aux études universitaires. Moins nantie, la CECM doit patienter jusqu'aux années 1920 avant d'inaugurer un cours postélémentaire, le primaire supérieur ${ }^{11}$.

9. Rapport financier du Bureau des commissaires d'écoles catholiques romains de la Cité de Montréal pour l'exercice 1898-1899, 5. Rapports financiers, Service des finances, ACSDM. Report of the Protestant Board of School Commissioners for Montreal, 1898-99, 11-12. AEMSB.

10. Protestant School Board of Greater Montreal. Annual Report, 1958-1959, 7-8. AEMSB. Rapport du trésorier de la Commission des écoles catholiques de Montréal, 1958-1959, 5-6. ACSDM.

11. À propos de l'histoire des high schools protestants au Québec au XIX ${ }^{\mathrm{e}}$ siècle, nous référons aux études suivantes: Gillian M. Burdett, The High School for Girls, Montreal, 1875-1914, mémoire de maîtrise (histoire), Université McGill, 1963, et Ann Drummond, From Autonomous Academy to Public High School: Quebec English Protestant Education, 1829-1889, mémoire de maîtrise (histoire), Université McGill, 1986. 


\section{LES DÉBUTS DE L'ENSEIGNEMENT SECONDAIRE PUBLIC FRANCOPHONE}

Le mode de répartition de la taxe scolaire ne peut seul être mis en cause dans l'émergence tardive d'une filière secondaire à la CECM. Depuis la dernière moitié $d u \mathrm{XIX}^{\mathrm{e}}$ siècle, les membres des professions libérales envoient leurs enfants dans les collèges classiques, des établissements privés prestigieux, qui les préparent à l'université et offrent, selon eux, un enseignement de qualité conforme à leur rang social. Bien servie par ces institutions qui agissent à titre d'instance de reproduction sociale, la bourgeoisie canadienne-française est peu pressée d'améliorer le financement des écoles publiques et encore moins d'y instaurer une filière secondaire ${ }^{12}$.

Au début $\mathrm{du} \mathrm{xx}^{\mathrm{e}}$ siècle, le gouvernement libéral de Lomer Gouin, en réponse aux transformations sociales engendrées par l'industrialisation, accrôit la participation financière de l'État dans le domaine de l'enseignement professionnel et supérieur. De nouvelles institutions, des écoles techniques et des facultés commerciales et scientifiques principalement, jaillissent dans le paysage scolaire. Ces institutions visent à former une main-d'œuvre qualifiée et à favoriser l'émancipation économique des Canadiens français ${ }^{13}$. La CECM ne se tient pas à l'écart d'un tel mouvement. Elle inaugure au début des années 1920 les premières classes "primaires supérieures » de $9^{\mathrm{e}}, 10^{\mathrm{e}}$ et $11^{\mathrm{e}}$ années. Elle fait écho aux revendications des libéraux, des hommes d'affaires, des congrégations enseignantes et des syndicats internationaux qui demandent avec insistance la création d’une filière éducative spécialement conçue pour les fils des classes

12. Pour davantage d'informations sur l'histoire et le développement des collèges classiques, voir Claude Galarneau, Les collèges classiques au Canada français (Montréal, Fides, 1978), 287 p.

13. Le gouvernement Gouin fonde en 1907 deux écoles techniques, puis l'École des Hautes Études commerciales en 1910. Il augmente les subventions de l’École Polytechnique fondée en 1873, qui crée à son tour en 1907 la section appelée à devenir plus tard l’École des Beaux-Arts. Le gouvernement encourage aussi l'ouverture de la Faculté des Sciences en 1920 à l'Université de Montréal. L’Université Laval, de son côté, crée une école d'arpentage et de foresterie respectivement en 1907 et 1910 et fonde l’École supérieure de chimie en 1920. Au sujet des développements éducatifs au Québec au début du $\mathrm{xx}^{\mathrm{e}}$ siècle, voir J.-P. Charland, Histoire de l'enseignement technique et professionnel (Québec, Institut québécois de recherche sur la culture, 1982), 482 p.; Pierre Dandurand et Marcel Fournier en collaboration avec Léon Bernier, «Développement de l'enseignement supérieur, classes sociales et luttes nationales au Québec», Sociologie et Sociétés, XII,2 (1980) : 101-131; Raymond Duchesne, «D’intérêt public et d'intérêt privé : l'institutionnalisation de l'enseignement et de la recherche scientifique au Québec (1920-1940)", dans Yvan Lamonde et Esther Trépanier, dir., L'avènement de la modernité culturelle au Québec (Québec, Institut québécois de recherche sur la culture, 1986), 189-230; Robert Gagnon, Histoire de la Polytechnique de Montréal (Montréal, Boréal, 1991), 526 p. ; Pierre Harvey, Histoire de l'École des Hautes études commerciales, 1 : 1887-1926 (Montréal, Québec/Amérique et Presses HEC, 1994), 382 p. 
moyennes et populaires qui les prépareraient à entrer dans les écoles techniques et dans les facultés commerciales et scientifiques nouvellement fondées ${ }^{14}$.

Toutefois, les élites canadiennes-françaises ne voient pas toutes d'un bon œil l'inauguration de cette filière éducative. Elles craignent que l'accès des classes moyennes et populaires aux études supérieures mine l'ordre social et que les écoles primaires supérieures puissent concurrencer les collèges classiques. Sans doute pour calmer ces appréhensions, le Comité catholique du Conseil de l'Instruction publique adopte, en 1929, un programme d'études qui exclut l'enseignement du latin de l'enseignement primaire supérieur. Or, à l'époque, la connaissance du latin constitue la condition d'entrée à la plupart des facultés de l'université. Le primaire supérieur prendra donc la forme d'un cours spécialisé, un prolongement de l'enseignement élémentaire, qui préparera ses élèves aux carrières des métiers spécialisés, des sciences, du commerce et du génie. Les collèges classiques, beaucoup moins accessibles en raison des droits de scolarité élevés qu'ils exigeaient bien souvent, continueront de constituer la voie royale conduisant aux facultés les plus prestigieuses comme la médecine, le droit et la théologie ${ }^{15}$.

En 1930, le Conseil de l'Instruction publique reconnaît au secteur anglais de la CECM un programme d'études distinct, inspiré de celui des high schools protestants qui comprend l'enseignement du latin et qui garantit aux élèves anglo-catholiques l'accès à l'ensemble des facultés ${ }^{16}$.

14. Pour le développement de l'enseignement primaire supérieur à Montréal, voir W. Johnston, L'école primaire supérieur et le high school public à Montréal (1920-1945), thèse de doctorat (histoire), Université de Montréal, 1992, 280-293 et R. Gagnon, Histoire de la Commission..., op. cit., 153-162. La question du rôle des congrégations enseignantes dans l'élaboration du primaire supérieur est abordée dans Paul-André Turcotte, L'enseignement secondaire public des Frères éducateurs (1920-1970): utopie et modernité (Montréal, Bellarmin, 1988), 220 p., et dans «Sécularisation et modernité: les frères éducateurs et l'enseignement secondaire public entre 1920 et 1970 ", Recherches sociographiques, 30,2 (mai-août 1989): 229-248; Georges Croteau, Les frères éducateurs, 1920-1965. Promotion des études supérieures, modernisation de l'enseignement public (Montréal, HMH, coll. "Cahiers du Québec - psychopédagogie», 1996), 193 p. Pour un regard comparatif avec l'Ontario, voir R. D. Gidney et W. P. J. Millar, Inventing Secondary Education. The Rise of the High School in NineteenthCentury Ontario (Montréal/Kingston, McGill-Queen’s University Press, 1990), 411 p.

15. Entre 1933 et 1953 , de $69 \%$ à $74 \%$ des revenus des collèges classiques proviennent des droits de scolarité, de $10 \%$ à $7 \%$ des subventions gouvernementales et environ $20 \%$ d'autres sources de financement comme les placements, les dons ou les emprunts, les activités extra-éducationnelles et les souscriptions diocésaines. L'Organisation et les besoins de l'Enseignement classique dans le Québec. Mémoire de la Fédération des collèges classiques à la commission royale d'enquête sur les problèmes constitutionnels (Montréal, Fides, 1954), 129.

16. Voir Robert Gagnon, Anglophones at the CECM (Montréal, CECM, 1996), 49-63, et Mélanie Lanouette, Penser l'éducation, dire sa culture. Les écoles catholiques anglaises au Québec, 1928-1964, mémoire de maîtrise (histoire), Université Laval, 2004. 
Ce n'est qu'en 1955 que les francophones disposeront véritablement d'un cours secondaire public, intégré aux études supérieures, avec la création des sections générale, commerciale, scientifique et classique ${ }^{17}$.

Enfin, rappelons que le primaire supérieur agira à titre d'instance de promotion sociale pour les classes moyennes et populaires qui ne pouvaient se payer des études dans les collèges classiques. Entre 1932 et 1945, le nombre d'écoles primaires supérieures passe de 172 à 382 et le nombre d'élèves de 4715 à 31 396. À titre de comparaison, la population des collèges classiques passe pour la même période de 9686 à 13404 élèves. Entre 1935 et 1940, 68 institutions d'enseignement classique sont fondées, dont 29 pendant la période qui s'étend de 1920 à $1939^{18}$.

\section{LE FINANCEMENT DES COLLÈGES CLASSIQUES ET DES ÉCOLES PRIMAIRES SUPÉRIEURES}

Force est de constater que le primaire supérieur, puis le secondaire public, resteront le parent pauvre de l'enseignement secondaire au Québec jusqu'aux années 1960. Cette préférence des élites canadiennes-françaises pour les collèges classiques se traduit aussi par un faible financement par l'État des écoles primaires supérieures et des écoles secondaires publiques. Ainsi, au lendemain de la Seconde Guerre mondiale, les subventions gouvernementales accordées aux collèges classiques surpassent celles distribuées aux écoles primaires supérieures malgré des inscriptions inférieures en nombre (tableau 1).

17. En 1923, le Comité catholique du Conseil de l'Instruction publique adopte un nouveau programme d'études. Auparavant, l'enseignement primaire était constitué de quatre années élémentaires, suivies de deux années modèles et de deux années académiques. À partir de 1923, l'enseignement primaire est constitué de six années élémentaires et de deux années primaires complémentaires ( $7^{\mathrm{e}}$ et $8^{\mathrm{e}}$ années). En 1929, le Comité catholique implante le cours primaire supérieur $\left(9^{\mathrm{e}}, 10^{\mathrm{e}}\right.$ et $11^{\mathrm{e}}$ années). Dix ans plus tard, le cours élémentaire est rallongé d’une année. Les $8^{\mathrm{e}}$ et $9^{\mathrm{e}}$ années deviennent le primaire complémentaire et les $10^{\mathrm{e}}, 11^{\mathrm{e}}$ et $12^{\mathrm{e}}$ années composent le primaire supérieur. Le nouveau programme d'études est divisé en deux sections pour les garçons (commerciale et scientifique) et trois pour les filles (générale, commerciale et spéciale). En 1955, les cours primaire complémentaire et supérieur prennent le nom de cours secondaire. Il renferme quatre sections: le cours général qui prépare au marché du travail ou aux écoles techniques; le cours commercial qui destine à des emplois dans le monde des affaires; le cours scientifique qui mène à des études collégiales et universitaires et la section classique qui correspond aux quatre premières années offertes dans les collèges classiques. Enfin, en 1969, le cours élémentaire n'est plus que de six années et le cours secondaire totalise cinq années. Voir R. Gagnon, Histoire de la Commission..., op. cit., 175-176, 207-208, 243-244.

18. G. Croteau, Les frères éducateurs..., op. cit., 96-97. L'Organisation et les besoins de l'Enseignement classique..., op. cit., 52-53. C. Galarneau, Les collèges classiques..., op. cit., 66-67. 
Tableau I

Comparaison entre la population scolaire des collèges classiques, des écoles primaires supérieures et des écoles secondaires publiques et la distribution des subventions gouvernementales en 1945 et en 1960

\begin{tabular}{|c|c|c|}
\hline & $\begin{array}{l}\text { Nombre } \\
\text { d'élèves }\end{array}$ & $\begin{array}{c}\text { Subventions } \\
\text { gouvernementales }(\$)\end{array}$ \\
\hline \multicolumn{3}{|l|}{1945} \\
\hline Collèges classiques & 13404 & 300000 \\
\hline Écoles primaires supérieures $\left(9^{\mathrm{e}}, 10^{\mathrm{e}}, 11^{\mathrm{e}}\right.$ années $)$ & 31396 & 75000 \\
\hline \multicolumn{3}{|l|}{1960} \\
\hline Collèges classiques & 30059 & 4123000 \\
\hline $\begin{array}{l}\text { Écoles secondaires } \\
\left(8^{\mathrm{e}}, 9^{\mathrm{e}}, 10^{\mathrm{e}}, 11^{\mathrm{e}}, 12^{\mathrm{e}} \text { années }\right)\end{array}$ & 217721 & 550000 \\
\hline
\end{tabular}

Sources: Rapport du Surintendant de l'Instruction publique pour la Province de Québec (RSIPPQ), pour les années 1945-1946 et 1960-1961. Mémoire de l'Université Laval à la Commission royale d'enquête sur l'enseignement, juin 1962, 86.

Par ailleurs, le partage des subventions gouvernementales entre les écoles primaires supérieures et les collèges classiques ne tient aucunement compte de l'expansion du primaire supérieur et de la progression de ses effectifs scolaires au lendemain de la Seconde Guerre mondiale. En effet, à la fin des années 1950, cette distribution des deniers publics ne correspond plus à la réalité sociale et éducative du Québec, alors que les effectifs scolaires du cours secondaire public sont nettement supérieurs à ceux des établissements d'enseignement classique.

Au tableau 1, on constate la volonté du gouvernement Duplessis (1944-1959) d'encourager l'enseignement privé moins coûteux pour les coffres de l'État et déjà financé partiellement par les droits de scolarité. Les écoles secondaires publiques n'accaparent, à la fin des années 1950, qu'à peine un dixième des subventions gouvernementales, même si leurs élèves représentent plus des quatre cinquièmes de la population inscrite au niveau secondaire ${ }^{19}$.

À Montréal, les effectifs des classes primaires supérieures, puis secondaires, progressent aussi très rapidement. Entre 1945 et 1961, le nombre d'élèves de la CECM inscrits aux cours primaire complémentaire $\left(8^{\mathrm{e}}\right.$ et $9^{\mathrm{e}}$ années) et supérieur $\left(10^{\mathrm{e}}, 11^{\mathrm{e}}\right.$ et $12^{\mathrm{e}}$ années $)$, puis au cours secondaire

19. Rapport du Surintendant de l'Instruction publique de la Province de Québec, 1959-1960, 10-11. (RSIPPQ). Mémoire de l'Université Laval à la Commission royale d'enquête sur l'enseignement, juin 1962, 86. 
( $8^{\mathrm{e}}$ à la $12^{\mathrm{e}}$ année) passe de 13715 à 40097 . Toutefois, le faible financement de l'État pour l'enseignement secondaire public et le mode de répartition de la taxe scolaire limitent l'accessibilité de la population francophone aux institutions d'éducation et entravent son processus de scolarisation. En effet, la CECM se trouve incapable d'offrir à sa clientèle scolaire les mêmes infrastructures d'enseignement secondaire que la PSBGM ${ }^{20}$.

\section{LES MOUVEMENTS PRÉCURSEURS}

À la faveur de la prospérité de l'après-guerre, on assiste à la montée d'une classe sociale, la nouvelle classe moyenne, qui détient principalement les emplois du secteur tertiaire. On inclut dans cette catégorie les emplois dans le transport et les communications, les services financiers, le travail de bureau, les secteurs de la vente, de la santé et de l'éducation, de l'administration publique et parapublique ${ }^{21}$. Les membres de ces nouvelles classes moyennes ont profité abondamment du développement du primaire supérieur, puis du secondaire public qui a permis à plusieurs d'entre eux de poursuivre leurs études dans les facultés des sciences, du commerce, du génie, de pédagogie et des sciences sociales ${ }^{22}$.

Ce nouveau groupe social diplômé et qualifié prend conscience de son influence grandissante dans la société et réclame un système d'éducation mieux adapté à ses aspirations sociales. Les membres de cette nouvelle

20. À titre de comparaison, en 1945, la PSBGM dispose de 13 high schools pour instruire 9243 élèves, tandis que la CECM possède seulement 4 écoles secondaires et deux high schools pour 13715 élèves. La majorité de ces derniers doivent suivre leurs cours dans des classes aménagées dans les écoles élémentaires. Protestant School Board of Greater Montreal, Annual Report, 1945-1946, 5. AMSB. Rapport du trésorier de la Commission des écoles catholiques de Montréal, 1945-1946, 8. Rapports financiers, Service des Finances, ACSDM.

21. En 1891, les travailleurs et les travailleuses œuvrant dans les secteurs de la vente et des services ne composent que le quart de la main-d'œuvre active au Québec contre 39\% en 1941, $43 \%$ en $1951,52 \%$ en 1961 et finalement $62 \%$ en 1971 . Voir Réjean Pelletier, Partis politiques et société québécoise. De Duplessis à Bourassa (Montréal, Québec/Amérique, 1989), 161-169; Gérald Fortin, La fin d'un règne (Montréal, Hurtubise HMH, coll. «Sciences de l'homme et humanisme», $\mathrm{n}^{\circ}$ 3, 1971), 397 p.; Jean-Louis Roy, La marche des Québécois. Le temps des ruptures (1945-1960) (Montréal, Leméac, 1976), 381 p.; Dorval Brunelle, La désillusion tranquille (Montréal, Hurtubise HMH, coll. «Cahiers du Québec », n 40, 1978), 77-81; Michael D. Behiels, Prelude to Quebec's Quiet Revolution: Liberalism versus Neo-Nationalism, 1945-1960 (Kingston/Montréal, McGill-Queen’s University Press, 1985), 366 p.

22. En 1952-1953, 38\% des étudiants des universités francophones du Québec ne sont pas des diplômés des collèges classiques. Leur proportion atteint $51 \%$ en 1960-1961. Ceux qui ne possèdent pas le baccalauréat ès art, décerné par les collèges classiques, se retrouvent principalement dans les facultés des sciences sociales, de commerce, de génie et sciences et de pédagogie. L'Organisation et les Besoins de l'Enseignement..., op. cit., 325. Procès-verbaux du Comité catholique (PVCC), 12 mai 1954, 17 avril 1961. 
classe moyenne sont représentés au sein des chambres de commerce, des syndicats, des mouvements coopératifs et des commissions scolaires. Ces organismes constitueront le fer de lance de la contestation sociale pour réclamer la démocratisation du système d'éducation. La CECM ne demeure pas en reste et s'impose dans l'après-guerre comme l'un des principaux porte-parole de la nouvelle classe moyenne. Les commissions royales d'enquête deviennent une tribune privilégiée pour faire valoir ses idées ${ }^{23}$. Lors de la Commission royale d'enquête sur les problèmes constitutionnels du Québec (1953-1954), plus connue sous le nom de Commission Tremblay, la CECM défend sa conception de l'éducation.

La CECM valorise une école publique «soutenue uniquement sinon en grande partie par le trésor public». Les revenus des commissions scolaires devraient être établis d'après les besoins réels des commissions scolaires plutôt qu'en fonction «du seul revenu de la propriété foncière» et «de la fortune ou le manque de fortune des parents de la foi religieuse que cette commission scolaire représente». La CECM préconise l'abolition de la taxe scolaire et l'octroi par les gouvernements provincial et municipal d'une partie des impôts sur le revenu et de la taxe de vente. Enfin, la CECM demande aussi que l'enseignement secondaire soit financé par le gouvernement provincial ${ }^{24}$.

Le rapport de la Commission royale d'enquête se montre favorable aux doléances des acteurs de l'éducation, notamment la CECM. Pour la première fois, le caractère discriminatoire du mode de financement scolaire et son incapacité à combler les besoins en éducation de la population francophone à Montréal sont reconnus :

Les modes de financement en vigueur, en 1950-51, ne permettaient pas de financer les écoles publiques même si celles-ci n’avaient qu’à prévoir les

23. En réponse à l'empiètement du gouvernement fédéral dans les champs de compétence provinciale après la Seconde Guerre mondiale, le gouvernement Duplessis met sur pied la Commission royale d'enquête sur les problèmes constitutionnels du Québec, en 1953. D’abord circonscrites principalement à la question de la fiscalité intergouvernementale, les interventions devant la Commission prennent la forme d'une vaste réflexion sociale, notamment sur les problèmes liés à l'éducation. De fait, 140 des 240 mémoires déposés portent sur les questions du financement scolaire, de l'accessibilité aux études supérieures, de la coordination du système scolaire et de la modernisation des structures scolaires. A. Tremblay, Le ministère de l'éducation..., op. cit., 49-58. Pour voir le rôle joué par la CECM dans la contestation du système scolaire entre 1945 et 1960, voir Jean-Philippe Croteau, «La CECM sous Duplessis : les racines de la contestation (1945-1960)", Bulletin d'histoire politique, 16,1 (automne 2007): 225-241.

24. Mémoire de la Commission des Écoles catholiques de Montréal à la Commission royale d'enquête sur les problèmes constitutionnels, 14. Commission royale d'enquête sur les problèmes constitutionnels. Commission royale d'enquête sur la situation scolaire au Québec (1954). ACSDM. 
dépenses occasionnées par la seule augmentation «naturelle» des populations scolaires et même si elles se contentaient de payer aux professeurs catholiques des salaires nettement inférieurs [...] À plus forte raison, ces modes de financement ne permettraient-ils ni d'augmenter le rendement pédagogique des écoles publiques ni d'améliorer les salaires des professeurs. [...] Voilà pourquoi si l'on admet la double nécessité d'augmenter la proportion des Canadiens français qui atteindront au niveau de scolarité correspondant à leurs aptitudes et de traiter les professeurs catholiques de la même façon que les protestants, il faut songer à de nouveaux modes de financement des écoles publiques ${ }^{25}$.

Le rapport final de la Commission Tremblay recommande que l'État accorde davantage de subventions aux commissions scolaires et qu'une partie de l'impôt sur le revenu et de la taxe de vente finance l'enseignement secondaire public. Il suggère aussi l'augmentation des subventions gouvernementales pour les collèges classiques et de l'allocation de bourses aux étudiants ${ }^{26}$. Toutefois, les membres de la Commission Tremblay n'osent pas remettre en question le mode de partage de la taxe scolaire basé sur l'appartenance religieuse des propriétaires. Pour eux, la propriété

\section{Graphique I}

Distribution en dollars du fonds d'éducation supérieur aux collèges classiques et aux écoles secondaires publiques entre 1945 et 1960 (années choisies)

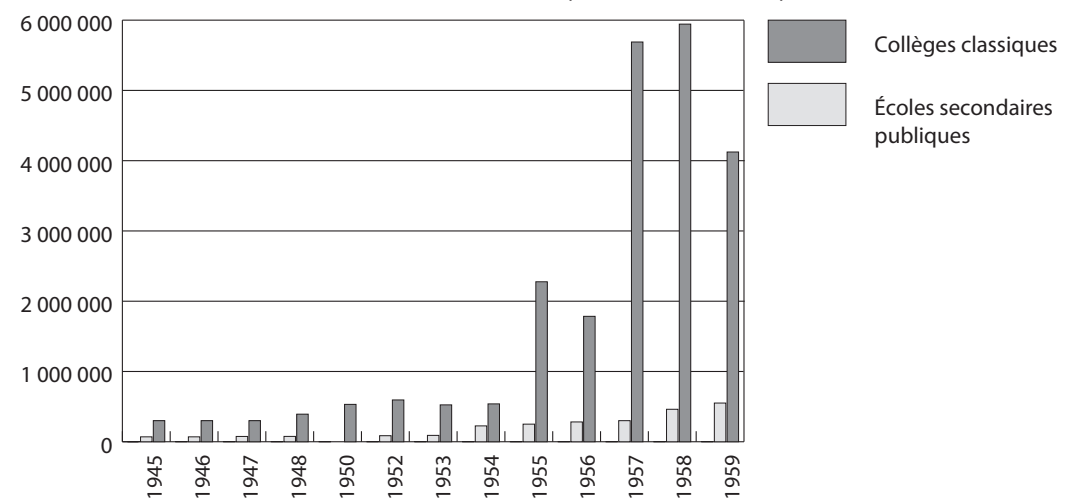

Sources: Rapport du Surintendant de l'Instruction publique pour la Province de Québec (RSIPPQ), 1945-1960

25. Arthur Tremblay, Contribution à l'étude des problèmes et des besoins de l'enseignement dans la province de Québec (Commission royale d'enquête sur les problèmes constitutionnels, 1955), Annexe 4175.

26. Rapport Tremblay, Tome 1, vol. 3, chap. 7 (Québec, 1956). Cité dans J.-L. Roy, La marche des Québécois...,op. cit., 248. 
foncière demeure le meilleur moyen «d'identifier le contribuable selon sa religion et assurer ainsi à chaque branche d'enseignement les revenus qui lui reviennent ${ }^{27}$ ». Cependant, comme on peut le voir au graphique 1 , les espoirs suscités pour les promoteurs du secondaire public par le rapport favorable de la Commission Tremblay ne se concrétiseront pas. L'augmentation des subventions gouvernementales pour les institutions postélémentaires profitera surtout aux collèges classiques.

\section{LA CECM ET LA RÉFORME DU FINANCEMENT SCOLAIRE}

Au début des années 1960, le personnel dirigeant de la CECM cède la place à une nouvelle équipe. Il faut rappeler qu'entre 1947 et 1973, le gouvernement provincial nomme quatre commissaires et l'archevêché de Montréal désigne les trois autres. Signe des temps nouveaux, le cardinal Léger choisit, en 1961, de nommer des laïques plutôt que des clercs. L'avocat Marc Jarry, ancien secrétaire de l'Université de Montréal, est désigné par le gouvernement libéral de Jean Lesage pour succéder au président de la CECM, Eugène Doucet, ami personnel de Duplessis et directeur du journal Montréal-Matin. C'est véritablement la relève de la garde à la CECM. Sous la gouverne d'Eugène Doucet, de 1949 à 1961, la CECM avait été associée aux forces conservatrices ${ }^{28}$. De plus, ces nouveaux commissaires sont recrutés en grande partie parmi les nouvelles classes moyennes ${ }^{29}$.

27. Ibid.

28. Robert Gagnon, “La CECM et la réforme Parent», Bulletin d'histoire politique, 12,2 (hiver 2004): 33-35. Entre 1846 et 1973, la CECM et la PSBGM ont connu respectivement dix et six modes de représentation des commissaires différents. Jusqu’à la Seconde Guerre mondiale, les commissaires de la CECM sont désignés par le gouvernement provincial, la ville de Montréal et l'archevêché. En 1947, le pouvoir de nommer les commissaires est retiré au Conseil municipal de Montréal. Entre 1846 et 1951, le gouvernement provincial et le Conseil de Ville nomment les commissaires de la Protestant Board School Commissioners of the City of Montreal (PBSCCM). Lors de la création de la PSBGM, en 1951, le gouvernement provincial et la ville de Montréal choisissent chacun quatre commissaires. Les autres commissions scolaires, membres de la PSBGM, désignent huit commissaires élus par les propriétaires fonciers. Les commissaires de la PBSCCM représentent donc entre 1951 et 1973 la moitié des membres de la PSBGM. Pour plus de renseignements sur le mode de représentation des commissaires de la CECM et de la PSBGM, voir R. Gagnon, Histoire de la Commission..., op. cit., 355-356, et MacLeod et Poutanen, Meeting of the People..., op. cit., 335-342.

29. Dans l'entre-deux-guerres, environ $70 \%$ à $80 \%$ des commissaires laïques de la CECM appartiennent à la classe d'affaires et aux professions libérales. Entre 1945 et 1960, près de la moitié des commissaires laïques sont issus des nouvelles classes moyennes. Jusqu'en 1960, les clercs composent entre $30 \%$ et $40 \%$ des nominations à la CECM. Enfin, nous avons relevé qu'au cours de la période suivante, de 1960 à 1973, les nouvelles classes moyennes représentent les deux tiers des commissaires. Entre 1960 et 1973, trois administrateurs, trois instituteurs, trois avocats, deux hommes d'affaires et deux syndicalistes siègent à la CECM. C'est sans compter la présence d'un 
La diversité du profil socioprofessionnel et scolaire des dirigeants de la CECM en fait de fervents promoteurs de l'enseignement secondaire public. Ils entendent bien profiter de la période de bouillonnement social qui s'annonce pour réclamer des changements dans la sphère éducative. Pour eux, l'époque où le secteur public constituait le parent pauvre du système scolaire est révolue. Il appartient désormais aux commissions scolaires d'éduquer la grande majorité des élèves:

L'école publique a longtemps été considérée comme le parent pauvre de nos structures scolaires et en a souffert; pauvre, elle l'était sûrement dans le monde d'hier où l'école des princes était la seule voie qui menait à l'enseignement supérieur. Les temps ont bien changé et il semble bien évident à ceux qui négocient des formules d'associations avec nos collèges que, de plus en plus, ce sont les commissions scolaires qui auront à assumer la responsabilité de préparer la grande majorité de nos jeunes à jouer pleinement le rôle que leurs talents individuels, développés au maximum, leur permettront d'assumer ${ }^{30}$.

L'un des thèmes récurrents du discours des dirigeants de la CECM est la nécessité pour les élèves francophones d'atteindre, particulièrement au secondaire, le même niveau de scolarisation que leurs camarades protestants. Cependant, pour ce faire, il importe de mettre la filière secondaire de la CECM sur un pied d'égalité avec celle de la PSBGM. Pour les commissaires, seule l'abolition d'un mode de financement discriminatoire mettra fin aux disparités entre les deux filières secondaires catholique et protestante. Il s'agit avant tout de corriger une erreur historique estime, à la fin des années 1960, André Gagnon, président de la CECM et successeur de Pierre Carignan:

Nous sommes héritiers d'un passé qui nous place devant une situation de fait. Depuis des années, les sommes investies par les protestants pour chaque élève sont beaucoup plus gigantesques que celles investies par les catholiques, de sorte que le réseau d'écoles protestantes est riche comparativement au réseau d'écoles pour les catholiques. Il faut assurer le rattrapage en ce domaine. L'école, importante pour le développement social, doit préparer des citoyens qui posséderont des moyens égaux sur le marché du travail ${ }^{31}$.

30. Livres des délibérations (LDC), 6 avril 1967. ACSDM.

31. LDC, 4 avril 1968. ACSDM. 
Il faut préciser que dans les années 1960, la CECM fait face à une progression fulgurante de ses effectifs. Entre 1963 et 1970, sa population scolaire atteint un sommet passant de 189972 à 228271 élèves. Néanmoins, en 1973, ce nombre diminue à 208679 élèves, une baisse causée en grande partie par le déclin de la clientèle du niveau élémentaire, alors que le secteur secondaire est en progression constante. Entre 1963 et 1973, les effectifs scolaires des élèves à l'élémentaire décroissent de 143976 à 115 130, tandis que ceux du secteur secondaire augmentent de 45996 à 93549 (voir tableau 2).

Tableau 2

Population des élèves de la CECM et de la PSBGM inscrits dans les classes élémentaires et secondaires en 1963 et en 1973

\begin{tabular}{l|c|c}
\hline & 1963 & 1973 \\
\hline Classes élémentaires & & 115130 \\
\hline CECM & 143976 & 38013 \\
\hline PSBGM & 42284 & \\
\hline Classes secondaires & 45996 & 93549 \\
\hline CECM & 22525 & 21381 \\
\hline PSBGM & & 208679 \\
\hline Total & 189972 & 59394 \\
\hline CECM & 64809 & \\
\hline PSBGM & &
\end{tabular}

Rapport financier, CECM, 1960-1973, Rapports financiers. Ressources financières, Archives de la Commission scolaire de Montréal (CSDM). Annual Report, PSBGM, 1960-1973, Archives English Montreal School Board.

Les effectifs scolaires de la PSBGM se stabilisent et amorcent un léger déclin durant cette période. En 1963, 42284 élèves fréquentent les écoles élémentaires et 21971 élèves sont inscrits dans les high schools. Au total, la PSBGM accueille 64255 élèves dans ses écoles. Ils ne sont plus que 59394 en 1973. Le nombre des inscriptions dans les écoles élémentaires et dans les high schools passe respectivement, entre 1963 et 1973, de 42284 à 38013 élèves et de 22525 à 21381 élèves (voir tableau 2) ) $^{32}$.

32. Protestant School Board of Greater Montreal. Annual Report (1963-1970). AEMSB. Cette explosion de la population scolaire est causée par l'instauration, en 1962, de la loi de l'instruction obligatoire à 15 ans révolus. De plus, le certificat de la $7^{\mathrm{e}}$ année ne dispense plus de la scolarité obligatoire. Enfin, le programme d'études comprend désormais un cours primaire de six années et un cours secondaire de cinq années. Toutes ces mesures ont provoqué l’afflux d'élèves aux écoles de la CECM. Par ailleurs, la croissance de la CECM s'inscrit dans un mouvement débuté au lendemain de la Seconde Guerre. En effet, la prospérité économique, la reprise de l’immigration, la migration 


\section{LA CECM ET LA RÉFORME DU MODE DE FINANCEMENT SCOLAIRE}

La CECM n'attend pas les recommandations finales de la Commission royale d'enquête sur l'enseignement (1960-1966) pour contester ouvertement les mécanismes du financement scolaire qui lui sont préjudiciables depuis près d'un siècle. À l'été 1963, elle demande au gouvernement que les taxes des compagnies et des sociétés soient désormais réparties selon le nombre d'enfants d'âge scolaire, entre 5 et 17 ans, plutôt qu'au prorata des populations catholique et protestante; une nouvelle formule de partage qui avantage la CECM, car ce groupe d'âge est plus fortement représenté chez les Canadiens français que chez les anglo-protestants en raison des familles plus nombreuses. Le gouvernement ménage la chèvre et le chou et vote une loi qui divise la taxe des compagnies commerciales et des sociétés, surnommée aussi la taxe des «neutres», d'après le nombre d'élèves qui fréquentent les deux commissions scolaires montréalaises. Cette formule de partage favorise légèrement la CECM, dont les effectifs scolaires sont en pleine croissance ${ }^{33}$.

Quelques mois plus tard, la CECM revient à la charge, mais avec un tout autre plan de partage de la taxe scolaire. Elle demande au gouvernement d'adopter un projet de loi - qui deviendra le projet de loi 139 - qui prévoit la mise en commun de tous les revenus des taxes des listes catholique, protestante et "neutre» prélevées sur l'île de Montréal et de les partager entre les commissions scolaires selon une formule pondérée de péréquation qui tiendrait compte du nombre d'élèves et du niveau d'enseignement. Le taux de la taxe scolaire des particuliers serait uniformisé à $1,10 \$$ par $100 \$$ d'évaluation foncière et celui des entreprises à $1,90 \$$ (voir tableau 3). Un même montant d'argent par élève serait fixé pour l'ensemble de l'île de Montréal. Lorsque les revenus des listes catholique et protestante seraient insuffisants pour respecter la somme d'argent qui a été prescrite pour chaque élève, la différence serait comblée par les taxes des compagnies et des sociétés ${ }^{34}$.

des populations rurales vers les villes et surtout le Baby Boom augmentent le nombre d'inscriptions à la CECM qui passe, entre 1945 et 1969, de 95000 à 173000 élèves. R. Gagnon, op. cit., 194-195 ; 244.

33. LDC, 18 juillet 1963. ACSDM.

34. Le montant alloué à chaque élève montréalais est calculé sous la forme d’unités. Ainsi, l'élève de l'élémentaire vaut une unité, celui de la maternelle 0,85 , celui des classes spéciales deux et celui du secondaire 1,75. Même si cela va à l'encontre du principe de l'équité financière défendu farouchement par la CECM, cette dernière accepte comme mesure de compromis que les noncatholiques et les non-protestants valent deux fois plus d'unités à chaque niveau d'enseignement que les catholiques et les protestants. Cet avantage concédé à la PSBGM, qui accueille dans ses 
Tableau 3

Taux de l'évaluation foncière par 100 \$à Montréal selon les listes des propriétés (1963-1973) (\$)

\begin{tabular}{l|c|c|c|c|c|c}
\hline Listes & 1963 & 1964 & 1965 & 1967 & 1969 & 1972 \\
\hline Catholique & 0,80 & 1,10 & 1,10 & 1,10 & 1,10 & 1,65 \\
\hline Protestante & 1,10 & 1,10 & 1,10 & 1,10 & 1,10 & 1,65 \\
\hline «Neutres» & 1,65 & 1,90 & 2,20 & 2,25 & 2,80 & 2,80 \\
\hline
\end{tabular}

Rapport financier, CECM, 1960-1973, Rapports financiers. Services des finances, Archives de la Commission scolaire de Montréal (CSDM). Annual Report, PSBGM, 1960-1973, Archives English Montreal School Board.

À l'instar de la CECM, la PSBGM a plaidé au lendemain de la Seconde Guerre mondiale pour une participation accrue de l'État au financement de l'enseignement secondaire public ${ }^{35}$. Toutefois, la PSBGM ne remet pas en cause le mode de répartition de la taxe scolaire. Même si les revenus disponibles provenant de la taxe scolaire demeurent insuffisants, il reste que sa situation financière est beaucoup plus enviable que la CECM. Elle se montre donc tout au long des années 1960 une partisane acharnée du statu quo.

Dans ces circonstances, la proposition de la CECM équivaut à une véritable déclaration de guerre. À l'automne 1964, la PSBGM monte aussitôt aux barricades contre ce projet qui risque d'amputer son budget de 3,5 millions de dollars. Les avocats de la PSBGM soumettent à la Commission Parent un mémoire qui se résume en deux points: que toutes les taxes scolaires des propriétaires non catholiques soient versées aux écoles protestantes et que les revenus des taxes des entreprises soient divisés au prorata des populations catholique et protestante comme c'était le cas par le passé.

On se souvient qu'en 1963 le gouvernement a adopté un nouveau mode de répartition des taxes des compagnies commerciales et des sociétés basé sur la fréquentation scolaire. La PSBGM demande donc de revenir à l'ancien mode de répartition de la taxe des «neutres». Rappelons aussi que seules les taxes scolaires des propriétaires juifs sont versées à la PSBGM.

écoles la grande majorité des élèves non catholiques et non protestants, est aboli en 1969. «Un vœu de la CECM. La péréquation s'étendrait à toute l'île de Montréal», La Presse, $1^{\text {er }}$ février 1969.

35. La PSBGM indique dans son mémoire que le coût par élève à l'école élémentaire Van Horne est de 185 \$, tandis que celui du West Hill High School est de 330 \$. The Protestant School Board of Greater Montreal. Brief for Submission to the Royal Commission of Inquiry on Constitutional Problems (1954), 4-5. 
Les propriétés des autres non catholiques et non protestants sont inscrites sur la liste des compagnies et des sociétés. La PSBGM considère que les revenus prélevés sur les propriétés des non-catholiques lui reviennent de droit, car elle s'occupe de l'éducation de la quasi-totalité de cette population $^{36}$.

La PSBGM envoie ses représentants à Québec pour plaider sa cause. Ils dénoncent le plan de partage de la CECM qui constituerait selon eux une grave entorse au principe de la confessionnalité et un coup de force porté contre les droits scolaires des protestants garantis par l'article 93 de l'Acte de l'Amérique du Nord britannique (AANB). Le président de la CECM, Marc Jarry, et la plupart des éditorialistes de la presse de langue française répliquent aux arguments de J. P. Rowat, président de la PSBGM, et l'accusent de «brouiller les cartes» lorsqu'il prétend que le projet de loi 139 renferme un danger pour la confessionnalité du système scolaire protestant.

De nombreux articles, notamment dans Le Devoir, tentent de démontrer que la réforme du mode de financement scolaire et la confessionnalité du système scolaire constituent deux problématiques complètement distinctes $^{37}$. Néanmoins, Jean Lesage est sensible aux revendications de la

36. Howard, Stalker \& Mc Dougall. 12 novembre 1964, 33-38. Taxes neutres. Taxes. Service des Finances. ACSDM. Entre 1870 et 1903, les Juifs avaient la possibilité d'inscrire leurs propriétés sur les listes catholique ou protestante. En 1903, la PSBGM - la PBSCCM à l'époque - négocie une entente avec la communauté juive qui est entérinée par une loi provinciale. La communauté juive est désormais considérée comme "protestante» à des fins financières et scolaires. Les propriétaires juifs paient leurs taxes aux écoles protestantes. Les élèves juifs possèdent les mêmes droits, devoirs et privilèges que les protestants. La PSBGM s'engage à dispenser de cours les élèves juifs lors de leurs fêtes religieuses et à les exempter des cours de morale chrétienne. La PSBGM a cherché à étendre cette loi à l'ensemble des non-catholiques et des non-protestants, ce qui lui a toujours été refusé par le gouvernement, même si la grande majorité de ces élèves fréquentaient ses écoles. Les propriétés non catholiques et non protestantes étaient inscrites sur la liste des compagnies commerciales et des sociétés. Pour la question scolaire juive, voir Pierre Anctil, Le rendez-vous manqué. Les Juifs face au Québec de l'entre-deux-guerres (Québec, Institut québécois de recherche sur la culture, 1988), 165-207; Arlette Corcos, Montréal, les Juifs et l'école (Sillery, Septentrion, 1997), 305 p. ; P. Anctil, «Ni catholiques ni protestants: les Juifs de Montréal», dans Tur Malka, Flâneries sur les cimes de l'histoire juive montréalaise (Sillery, Septentrion, 1997), 25-35; R. MacLeod et M. Poutanen, Meeting of the People..., op. cit., 195-223 ; Jean-Philippe Croteau, «Les immigrants et la Commission des écoles protestantes du Grand Montréal (1864-1931)", dans Jean-Michel Lacroix et Paul-André Linteau, Vers la construction d'une citoyenneté canadienne (Paris, Presses Sorbonne Nouvelles, 2006), 31-47.

37. En juillet 1964, Le Devoir consulte - sans le nommer - un avocat spécialisé en matière scolaire pour s'enquérir de la constitutionnalité de la loi 139. Celui-ci rappelle les trois points suivants: 1) À l'origine, les commissions scolaires puisaient dans un fonds commun et la répartition des revenus scolaires s'établissait en fonction de la population catholique et protestante. 2) Entre 1846 et 1868 , il n'existait pas de taxe scolaire. C'est la municipalité de Montréal qui, à même ses 
PSBGM. Il n'ignore pas le poids électoral de la minorité anglophone, surtout à Montréal. Il propose un amendement au projet de loi 139. Au lieu d'abaisser de $23 \%$ à $17 \%$ la part de la taxe des compagnies commerciales et des sociétés perçue par la PSBGM comme le souhaite la CECM, il suggère de la limiter à $22 \%$. La PSBGM ne subirait qu'une perte financière de $800000 \$$ au lieu de 3,5 millions $^{38}$.

La CECM dispose d'un allié de taille en la personne du ministre de l’Éducation, Paul Gérin-Lajoie, qui pilote personnellement le projet de loi. Gérin-Lajoie ne cache pas son intention d'en arriver progressivement à une péréquation des revenus de la taxe scolaire sur l'île de Montréal. Quelques jours plus tôt, Le Devoir avait appuyé sans réserve la CECM par la voix de son directeur, Claude Ryan, qui dénonçait l'usage abusif que faisait la PSBGM des droits confessionnels au mépris des principes de justice et d'équité :

Il faut d'abord parler de justice et d'égalité pour tous. En matière de fiscalité et de dépenses publiques, il faut toujours mettre au premier plan l'égalité des efforts et la parité des services. Tout le reste, droits acquis, privilèges particuliers, considérations de confessionnalité doit venir en second lieu. Le législateur ne doit pas se laisser fléchir par des arguments spécieux qui sous prétexte de sauver les droits acquis feraient bon marché de l'équitée ${ }^{39}$.

Face aux protestations de ses ministres les plus influents et de la presse de langue française, Lesage parvient habilement à trouver une échappatoire. Il refuse de prendre une décision définitive en prétextant que la Commission royale d'enquête sur l'éducation n'a pas encore remis son rapport final. Le ministre de l'Éducation, Paul Gérin-Lajoie, propose un compromis temporaire visant à satisfaire la PSBGM et la CECM. Le gouvernement retient presque intégralement la formule de partage proposée par la CECM et insère trois modifications. D'abord, la PSBGM obtient la garantie qu'elle recevra au moins $20 \%$ des revenus de la taxe des "neutres». Ensuite, cette loi ne s'applique que pour l'année courante de 1964-1965. Enfin, le

revenus, finançait les commissions scolaires. 3) Les trois listes confessionnelles - catholique, protestante et «neutre» - ont été établies à la demande des protestants. Avant cette date, les revenus des commissions scolaires étaient puisés à un fonds commun. C'est ce fonds commun que la CECM veut restituer. Enfin, l'avocat conclut: «L'AANB protège la confessionnalité des écoles (catholiques et protestantes), mais pas la taxation. Il ne faut pas confondre taxation et confessionnalité». «Le Bill 139 viole-t-il l’AANB?», Le Devoir, 15 juillet 1964.

38. "Lesage suggère que la contribution du PSBGM à la péréquation soit diminuée à 800000 \$», Le Devoir, 17 juillet 1964.

39. Claude Ryan, «De la légalité à l'équité», Le Devoir, 14 juillet 1964. 
gouvernement s'engage à présenter dans un an un nouveau projet de loi à la lumière des recommandations de la Commission Parent. C'est la CECM qui bénéficie principalement du nouveau partage puisqu’elle reçoit les trois quarts du montant de la taxe des compagnies commerciales et des sociétés remis aux commissions scolaires catholiques (voir graphique 2$)^{40}$.

\section{Graphique 2}

Montant en dollars des taxes des compagnies et des sociétés partagées entre la PSBGM et la CECM

(1962-1973)

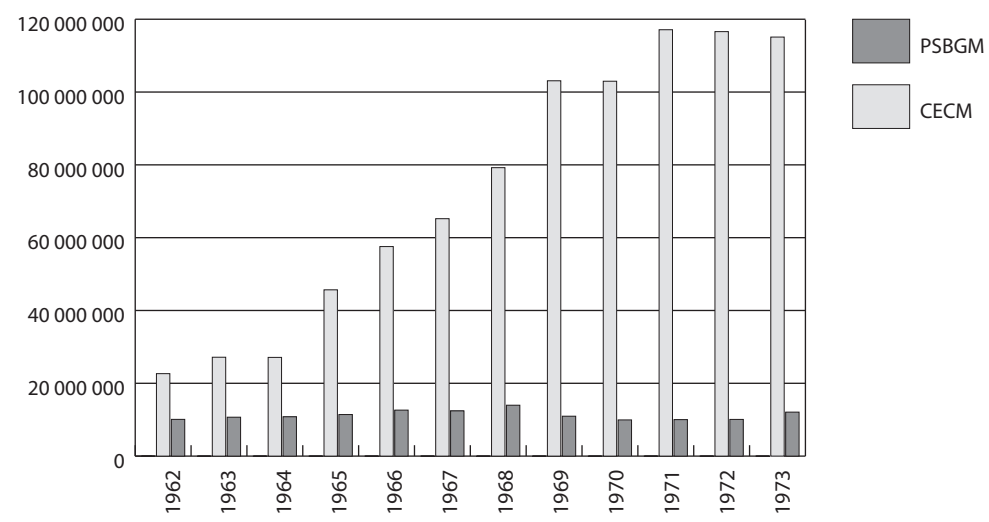

Sources: Rapports financiers (1963-1973). Rapports financiers. Service des Finances. ACSDM. Annual Report (1963-1973). AEMSB.

L'année suivante, le gouvernement renouvelle la loi, mais accorde cette fois à la PSBGM une part de $18 \%$ des revenus de la taxe des «neutres». Loin d'acquiescer au principe de la péréquation, la PSBGM soumet à l'Assemblée législative le projet de loi 190 qui prône un retour à l'ancien mode de répartition de la taxe des «neutres», soit au prorata des populations catholique et protestante. L'avocat de la PSBGM, T. P. Palmer, subit les foudres du premier ministre Jean Lesage, qui, cette fois, s'est rallié à la cause de la CECM sans doute à des fins électoralistes. Il rabroue publiquement la PSBGM pour avoir osé, selon lui, soumettre un projet de loi aussi inéquitable, alors que les minorités francophones de la Saskatchewan luttent pour faire reconnaître leurs droits scolaires les plus élémentaires.

40. "Taxe scolaire: péréquation catholique et protestante au Québec», Le Devoir, 18 juillet 1964. 
Néanmoins, pour apaiser la PSBGM, le taux de la taxe des compagnies et des sociétés est haussé de 1,90 à 2,20\$(voir tableau 3$)^{41}$.

En 1966, la CECM suggère au gouvernement d'abaisser la part de la PSBGM à $16 \%$. Cette dernière écrit au ministre de l'Éducation sous le gouvernement de l'Union nationale, Jean-Jacques Bertrand, pour faire savoir sa désapprobation. Une telle mesure imposerait un déficit à la commission scolaire et risquerait de dégrader la qualité de l'enseignement offert dans les écoles protestantes:

The P.S.B.G.M. recognizes the need to distribute school taxes in a manner which will provide equality of educational opportunity for each child in Quebec, regardless of religion. However, any idea that equality of opportunity and revenue go side by side, hand in hand, and are natural concomitants presupposes that both the Catholic system and the Protestant system are at the same state of development. This is not the case at the present. Without arguing the merits or the demerits of the formula advanced in the last two years by the C.E.C.M. (generally referred to as "perequation Formula"), it is the contention of the P.S.B.G.M. that its immediate application, in toto, would only result in surplus revenue for the C.E.C.M. and a staggering deficit for the P.S.B.G.M. or a severe cut-back which would vastly reduce the educational standards of the P.S.B.G.M. The P.S.B.G.M. regards this latter possibility as a distinctly retrograde step and one which they sincerely believe would not be in the best interests of the Province as a whole ${ }^{42}$.

La protestation de la PSBGM provoque la fureur de la CECM. Pour elle, les ententes de 1964-1965 et de 1965-1966 constituaient une première étape vers la généralisation progressive du principe de péréquation. Elle rappelle que chaque enfant "a droit à une éducation égale sans tenir compte de sa race, de sa langue, de sa religion ou de sa couleur». Elle maintient aussi que «l'éducation est un devoir social et public qui doit être à la charge de tous les contribuables sans tenir compte de sa race, de sa langue, de sa religion ou de sa couleur».

Dans un document envoyé au ministre de l'Éducation, elle tente de mettre en pièces l'argument de la PSBGM qui s'oppose au principe de la

41. "Compromise Reached in Quebec, On Sharing Neutral Panel Taxation», The Gazette, 4 juin 1965.

42. The Protestant School Board of Greater Montreal. Re: Problems presently confronting The Montreal Catholic School Commission and The Protestant School Board of Greater Montreal in connection with school taxation for the year 1966-67 and the disposition of Private Bills intended to regulate this matter but not yet presented to the legislature. June 28th, 1966. Taxes neutres. Taxes. Service des Finances. ACSDM. 
péréquation pour préserver la qualité de ses services éducatifs et de son enseignement:

On allègue que la P.S.B.G.M. a donné une meilleure éducation à ses élèves dans le passé et, partant, on veut en tirer l'argument qu'il lui faut plus d'argent pour continuer cette meilleure éducation. Quelle réponse pouvons-nous apporter à nos enfants qui demanderaient (et qui ont droit à) une éducation égale si on ne peut la leur offrir parce qu'on a donné (et qu'on continuerait à donner) une part plus importante des fonds aux autres, depuis des décennies, et que cela a contribué à créer la situation existante jusqu'à aujourd'hui? Nos enfants serontils bien fondés à croire à l'égalité au Canada, en matière d'éducation? Ne seront-ils pas amenés à croire qu'ils ne sont pas Canadiens à part entière? Qui pourra leur expliquer qu'en 1967, après 100 ans de Confédération, ils doivent recevoir moins pour leur éducation, parce qu'il en a été ainsi depuis $1869^{43}$ ?

Le gouvernement finit par reconnaître le principe de la péréquation malgré la résistance de la PSBGM et il réduit annuellement sa part des revenus des taxes des compagnies et des sociétés. Entre 1962 et 1970, cette dernière voit sa part passer de 31 à $9 \%$. Pour empêcher une baisse trop radicale de ses revenus, le taux de la taxe des «neutres » est haussé à 2,25\$ par $100 \$$ d'évaluation foncière en 1967 et à 2,80 \$, en 1969 (voir tableau 3 et graphique 2).

À la fin des années 1960, la PSBGM ne s’est toujours pas ralliée au principe de la péréquation et continue à dénoncer la diminution de sa part des revenus de la taxe des «neutres». En 1969, Peter Millar, commissaire à la PSBGM, écrit à S. F. White, directeur du service des finances au ministère de l’Éducation, pour lui signifier son opposition au principe de péréquation:

$[\ldots]$ we believe that despite its apparent equity it is really inequitable. If our system and that of the CECM were just starting up, there might well be an argument for spending equal dollars per child, but this is not the case. Over the years, we have build up a corps of well qualified teachers, many of them with degrees - on the Catholic side the ratio of degree teacher to children is much lower, therefore, our instructional costs are higher. This is only one of several examples of how unfair the application of perequation formula would be. The application of the method of distribution of Neutral tax as proposed in this Bill would bring our share of the tax almost to the vanishing point. This position, we are not prepared to accept ${ }^{44}$.

43. Mémoire de la Commission des écoles catholiques de Montréal au Ministre de l'éducation à la suite du mémoire du Protestant School Board of Greater Montreal du 11 octobre 1966. Taxes neutres. Taxes scolaires. Service des Finances. ACSDM.

44. 24 février 1969. Minute Book, PBSC. AEMSB. 
White lui répond que dorénavant le gouvernement distribuera les subventions en veillant à ce que les dépenses des commissions scolaires soient contrôlées et uniformisées sur une base équitable. Les principes défendus par la PSBGM sont dépassés, affirme White, «It therefore seems to us that the time has come to adopt for both Montreal Boards the system of financing that now applied to all other boards in the province ${ }^{45}$."

Peu à peu, on assiste à l'émergence d'une conception de l'éducation au sein de la communauté protestante qui tend à reconnaître la justesse d'un mode de péréquation. D’autant plus que la nouvelle distribution des taxes scolaires en faveur des commissions scolaires catholiques n'entraîne pas une diminution radicale des revenus de la PSBGM qui peut continuer à offrir des services éducatifs de même qualité dans ses écoles. Le partage de la taxe des compagnies et des sociétés, en 1964-1965, qui fixe un plafond de $20 \%$ à la PSBGM est bien accueilli par la presse de langue anglaise qui rappelle que les développements récents des commissions scolaires, notamment l'expansion du secteur secondaire et l'instauration de services spécialisés, nécessitent un nouvel arrangement financier:

Fortunately, there is no reason to believe that the Government intends to fall back on makeshift arrangements. There may be confidence that it is looking forward to a larger, sounder, and more lasting adjustment, in order that its declared sense of the importance of education, and of raising standards already expressed in principle, will have its justification in practice. There can be no solution in raising some standards only to lower others: the only real solution is to raise all $^{46}$.

Enfin, en 1972, se joue le dernier acte de la démocratisation du financement scolaire commencé dix ans plus tôt. Le gouvernement vote la loi 71 qui crée le Conseil scolaire de l'île de Montréal (CSIM). La question du partage des ressources financières entre les diverses commissions scolaires est réglée. Les pouvoirs du CSIM comportent, entre autres, la tâche d'assurer le financement des commissions scolaires, de veiller à la planification de leur développement et à l'organisation des services communs $^{47}$.

45. 3 mars 1969. Minute Book, PBSC. AEMSB.

46. «School Tax Problems», The Gazette, 7 juin 1965. Le Montreal Star tient un discours similaire: "The solution in the best interest of Montreal's school children is equal per-pupil taxation revenues. This would be keeping with the principle equal educational opportunity for all, as advocated by the Department of education and Parent Commission". "Temporary Solution. Compromise on School Taxes ", Montreal Star, 6 juin 1965.

47. Ibid., 250-253. 
D’après Gérard Barbeau, premier directeur général du CSIM, il y a tout lieu de se réjouir, car la création de cet organisme représente une réelle mesure de progrès:

Le $1^{\text {er }}$ juillet 1973 marquera donc le début d'une nouvelle ère dans la vie scolaire métropolitaine qui se trouve davantage placée sous le signe de la démocratisation à maints égards. D’une part, tous les commissaires auront été élus au suffrage universel. D'autre part, et ceci est encore plus important, les nouvelles structures scolaires permettront à l'ensemble des élèves de l'île de recevoir de meilleurs services d'éducation, grâce en partie à l'égalisation de l'effort fiscal de tous les citoyens et à une répartition des fonds disponibles en fonction des besoins des enfants et non plus en fonction de la richesse relative de la ville où ils demeurent ${ }^{48}$.

\section{UNE RÉVOLUTION DITE «TRANQUILLE »?}

La refonte du mode de financement scolaire amorcée à Montréal sous l'impulsion de la CECM a constitué une véritable "révolution» qui a mis fin aux disparités des revenus entre les commissions scolaires à Montréal, puis sur l'île de Montréal et enfin, qui a servi d'exemple à travers la province. Cette réforme financière pourrait se qualifier de «tranquille» dans la mesure où le gouvernement a réussi, en coopération avec la CECM, à procéder à une redistribution des fonds destinés à l'éducation sans provoquer un trop grand ressac au sein de la communauté anglophone. Par ailleurs, elle a atteint son objectif de départ, soit donner le coup d'envoi au développement de la filière secondaire des francophones en retard sur celle de la PSBGM.

Le gouvernement du Québec a choisi de recourir à une redistribution des revenus de la taxe scolaire de manière progressive en réduisant la part de la PSBGM prélevée sur les compagnies commerciales et des sociétés principalement au profit de la CECM. La part de la taxe des compagnies et des sociétés perçue par la PSBGM passera, entre 1962 et 1973, de 31 à $9 \%$ (voir graphique 2 ).

Pour compenser les pertes de la PSBGM, le gouvernement augmente subséquemment les taux d'imposition de la taxe des compagnies commerciales et des sociétés. La taxe des compagnies et des sociétés est augmentée au cours de la même période de 1,65 à 2,80 \$ par 100\$ d'évaluation

48. "Un bulletin d'information à l'intention des commissions scolaires de leur personnel et des parents ", Le Bulletin du Conseil de l'T̂le de Montréal, I,1, mai 1973. Conseil scolaire de Montréal, Bulletin du Conseil scolaire de Montréal de l'̂̂le de Montréal (1973-1974). Système scolaire. Commissions scolaires locales. Commissions scolaires. Diverses généralités. ACSDM. 
foncière, tandis que celle des catholiques passe de 0,80 à $1,65 \$$ par $100 \$$ et celle des protestants de $1,10 \$$ à $1,65 \$$ par $100 \$$ (voir tableau 3 ). La modification du mode de partage des taxes des «neutres», accompagnée de la hausse successive du taux d'imposition de la propriété financière, font pleuvoir sur la CECM une véritable manne financière. En 1963 et 1973, la CECM récolte respectivement 26 millions et 119 millions de dollars de la taxe scolaire. Au cours de cette période, les revenus de la taxe scolaire augmentent aussi pour la PSBGM, passant de 19 à 41 millions de dollars, une proportion beaucoup plus modeste que pour la CECM.

Dans les années 1960, le gouvernement provincial tente d'encourager la diffusion de l'enseignement secondaire parmi la population francophone en augmentant les subsides aux commissions scolaires catholiques. Le montant des subventions gouvernementales pour la CECM passe de 21 à 86 millions de dollars entre 1960 et 1973. La part des subventions gouvernementales compte pour $39 \%$ de ses recettes en 1973 contre $27 \%$ en 1960. Ces sommes considérables visent à rattraper le retard de cette commission scolaire au niveau des infrastructures et des services éducatifs offerts à la collectivité francophone, mais aussi à répondre à l'évolution de ses inscriptions en pleine croissance, notamment au secteur secondaire ${ }^{49}$. Tandis que l'État québécois déverse des millions de dollars dans les coffres de la CECM, il fait plafonner ses subventions à la PSBGM, ce qui réduit, entre 1960 et 1973, la proportion des aides gouvernementales sur ses revenus totaux de 28 à $14 \%$. Toutefois, en raison de la stabilité de la population scolaire de la PSBGM, celle-ci ne se trouve donc pas avec moins de revenus par élève (voir tableau 2 et graphique 3).

L'égalité financière entre les deux commissions scolaires constituera un long, mais inexorable processus réalisé grâce au concours de l'État et à la refonte du mode de partage de la taxe scolaire. Ce n'est qu'en 1973 que la CECM dépensera le même montant que la PSBGM. En 1960-1961, la CECM dépense 277 \$ par élève contre 382 \$ pour la PSBGM. Dix ans plus tard, la première débourse 718 \$ et la seconde, 804 \$. Enfin, la CECM rattrape la PSBGM l'année de la création du Conseil scolaire de l'Île de Montréal, en 1973. La CECM dépense 1009 \$ par élève et la PSBGM vient tout juste derrière avec 1006 (voir graphique 4).

49. La Commission des écoles catholiques de Montréal. Rapport du trésorier (1962-1973). Rapports financiers. Service des Finances. Services. ACSDM. 


\section{Graphique 3}

Montant en dollars des subventions gouvernementales et des taxes scolaires distribuées à la CECM et à la PSBGM

(1963-1973)

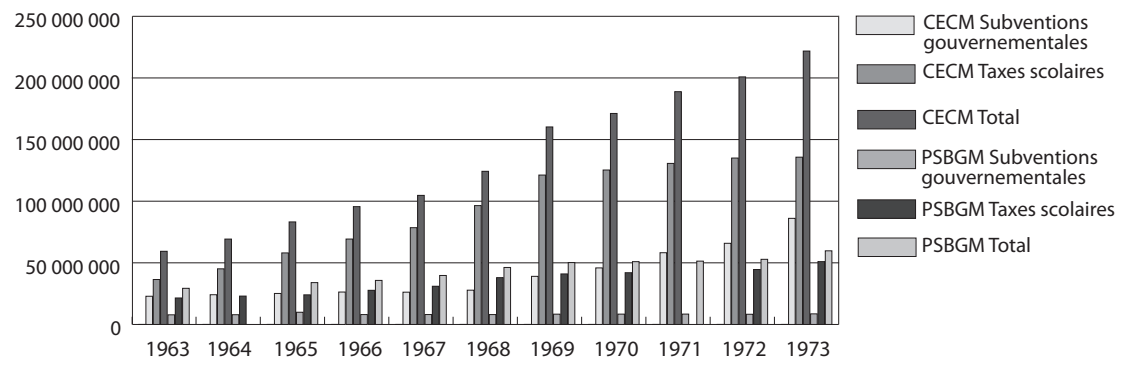

Sources: Rapports financiers (1963-1973). Rapports financiers. Service des Finances. ACSDM. Annual Report (1963-1973). AEMSB.

Graphique 4

Montant en dollars dépensé par élève par la CECM et la PSBGM (I96I-1973)

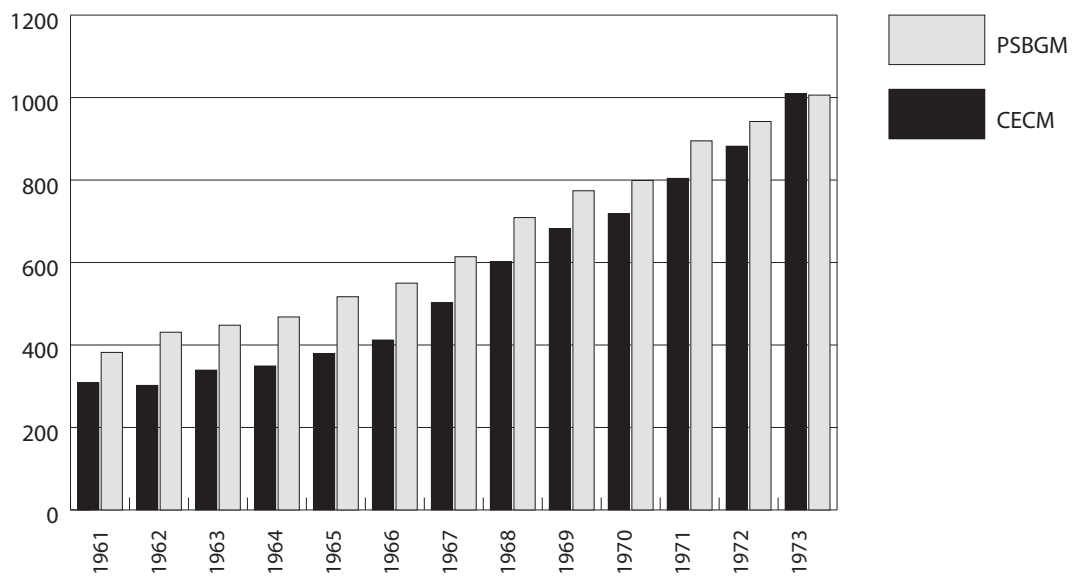

Sources: Rapports financiers (1963-1973). Rapports financiers. Service des Finances. ACSDM. Annual Report (1963-1973). AEMSB. 


\section{CONCLUSION}

Enfin, cette réforme a-t-elle porté ses fruits et permis aux francophones d'avoir le même accès à l'enseignement secondaire que leurs camarades anglo-protestants? Au lendemain de la Seconde Guerre mondiale, le secteur secondaire de la CECM était extrêmement marginal: à peine $10 \%$ de sa population totale le fréquentait contre le quart à la PSBGM. Dans le milieu des années 1960 , la proportion atteint $25 \%$ à la CECM, puis au début des années 1970 près de $45 \%$, surpassant même les protestants à partir de 1968. Pour ces derniers, cette proportion passe, entre 1950 et 1970, de 26 à $36 \%$. La proportion des effectifs des anglo-catholiques inscrits dans les high schools se stabilise à $20 \%$. Une proportion qui se rapproche des élèves de la PSBGM. Il faut rappeler que les élèves anglo-catholiques ont bénéficié d'un accès à une filière secondaire coordonnée aux études supérieures dès les années 1930, soit une génération avant les francophones. Néanmoins, à partir de 1968, la proportion d'élèves francophones inscrits au niveau secondaire équivaut à celui des anglo-catholiques et des protestants (voir graphique 5$)^{50}$.

\section{Graphique 5}

Pourcentage des élèves des secteurs français et anglais de la CECM et de la PSBGM qui fréquentent les écoles secondaires et les High Schools sur les inscriptions totales

(195I-197I)

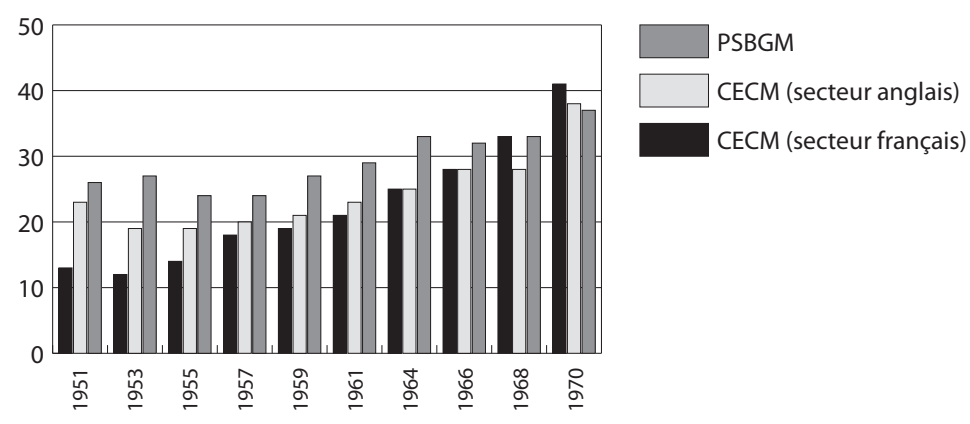

Sources: Statistiques (1951-1971). Services de la statistique. ACSDM. PSBGM. Annual Report (1951-1970). AEMSB.

50. Statistiques (1951-1971). Services de la statistique. Services. ACSDM. PSBGM. Annual Report (1951-1971). AEMSB. 
Notre analyse pourrait être complétée par une étude comparative sur le rapport entre le financement scolaire et l'éducation secondaire au Québec et en Ontario. À l'instar du Québec, un mode de financement scolaire basé sur le prélèvement d'une taxe scolaire sur les propriétés foncières a prévalu en Ontario. Les revenus de la taxe scolaire étaient répartis entre les écoles publiques et les écoles séparées dites «catholiques» d'après l'appartenance religieuse des propriétaires. Cependant, le gouvernement de l'Ontario a refusé jusqu'en 1985 de subventionner des écoles secondaires catholiques.

Dans ces conditions, la diffusion de l'enseignement secondaire catholique a relevé des institutions privées qui n'étaient pas subventionnées par l'État. Comment les élites éducatives catholiques de l'Ontario ont-elles réussi à surmonter ces obstacles financiers et assurer aux enfants de la minorité catholique l'accès à l'éducation secondaire et supérieure, ainsi qu'à la promotion sociale? Une telle étude pourrait démontrer que le rapport entre le financement scolaire et l'éducation secondaire ne se limite pas spécifiquement au Québec et qu'il a constitué un enjeu éducatif important dans plusieurs provinces, notamment l'Ontario, ainsi qu'un débat récurrent, de la fin du $\mathrm{XIX}^{\mathrm{e}}$ siècle jusqu'à récemment. Enfin, elle révélerait l'importance des liens entre la confessionnalité, l'éducation et le financement scolaire au sein des sociétés nord-américaines. 